\title{
ALIANZAS PÚBLICO PRIVADAS: UNA ALTERNATIVA PARA LA CO-FINANCIACIÓN DE OBRAS PÚBLICAS EN EL SECTOR DEL AGUA Y SANEAMIENTO URBANO EN ECUADOR
}

\author{
PRIVATE PUBLIC ALLIANCES AN ALTERNATIVE \\ FOR THE CO-FINANCING OF PUBLIC WORKS IN THE WATER \\ AND URBAN SANITATION SECTOR IN ECUADOR
}

Pablo Ramiro Chafla Martínez

\begin{abstract}
Resumen
Introducción La búsqueda de mecanismos nuevos y creativos de co-financiación de obra pública local, principalmente en el sector de agua y saneamiento, resultaría un ejercicio prioritario en Ecuador dado el actual contexto de crisis económica que sufre el país como consecuencia del desplome del precio de la principal mercancia (commodity) de exportación como es el caso del petróleo y el pago de las obligaciones de la deuda externa que han reducido sustancialmente los ingresos fiscales del pais y por ende la capacidad de inversión en obra pública. Objetivo. El objetivo central de esta investigación es presentar alternativas reales de inversión y financiación de obra pública municipal en la actual coyuntura económica en la que los Gobiernos Autónomos Descentralizados (GAD) Municipales deben hacer frente a la dotación de infraestructura pública, principalmente, con recursos propios y sin mayores aportes del Gobierno central. Método. La metodologia utilizada es el análisis el contenido de los modelos de co-financiación, gestión y administración de servicios públicos de agua y saneamiento como son las Alianzas Público-Privadas (APP), se pueden analizar como una alternativa viable para garantizar el derecho humano al agua y a un medio ambiente sano que contempla la constitución del país. Resultados. Las APP son viables como una medida excepcional de colaboración entre el sector público y privado para el sector de agua y saneamiento.
\end{abstract}

\section{Palabras clave}

Política pública, desarrollo de políticas públicas sectoriales, acuerdos público-privados, provisión de bienes públicos y privados, bienestar.

\begin{abstract}
Introduction. The search for new and creative mechanisms for co-financing of local public works, mainly in the water and sanitation sector, would be a priority exercise in Ecuador given the current context of economic crisis in the country as a result of the collapse of the price of the principal export commodity such as oil and the payment of external debt obligations that have substantially reduced the country's fiscal income and therefore the capacity for investment in public works. Objective. The main objective of this research is to present real alternatives for investment and financing of municipal public works in the current economic situation in which the Municipal Decentralized Autonomous Governments GAD must deal with the provision of public infrastructure mainly with their own resources and without major contributions from the Government Central. Method. The methodology used is content analysis of the models of co-financing, management and administration of public water and sanitation services, such as Public-Private Partnerships (PPP), can be analyzed as a viable alternative to guarantee the human right to water and a healthy environment that contemplates Constitution of the country. Results. PPP are viable as an exceptional measure of collaboration between the public and private sector for the water and sanitation sector.
\end{abstract}

\section{$\underline{\text { Keywords }}$}

Public policy, public sector policy developing, public-private agreements, provision of public and private goods, well-being.

JEL: H7-H76 


\section{Introducción}

La insuficiencia de recursos económicos para cubrir las cada vez mayores necesidades de financiación de obra pública a nivel local, se ha convertido en un desafío para los gobiernos locales, y con mayor razón si cabe, en las regiones de menor desarrollo, es por este motivo que la presente investigación plantea como objetivo principal presentar alternativas reales de inversión y financiación de obra pública municipal.

Dado el actual contexto de ralentización económica que sufre la economía ecuatoriana como consecuencia del desplome del precio de la principal mercancía (commodity) de exportación como es el caso del petróleo, el pago de las obligaciones de deuda externa, la baja recaudación y evasión tributaria, los numerosos casos de corrupción suscitados en las últimas décadas y los ajustes fiscales necesarios para equilibrar las cuentas públicas, han reducido sustancialmente los ingresos fiscales del país y por ende la capacidad de inversión en obra pública.

La necesidad de impulsar el crecimiento económico de América Latina ha llevado a varios de los gobiernos de la región a promover ambiciosos planes de desarrollo de infraestructuras principalmente públicas. En este marco, países como Brasil, México, Colombia, Perú, Chile y, recientemente, Ecuador están acometiendo en la actualidad diferentes planes inversores. Lograr acceder a modelos de Alianzas Público-Privadas (APP) o Participación Público-Privado (PPP) como también se las conoce, es una alternativa posible que contemplan los gobiernos de estos países para poder contar con el financiamiento y los recursos necesarios para mejorar su desarrollo (Banco de Desarrollo, 2015).

El análisis de esquemas alternativos de co-financiación de obra pública local, tanto de nueva construcción, como para la modernización de las infraestructuras que van en- vejeciendo (especialmente crítico en Latinoamérica donde el estado de deterioro de las infraestructuras es más grave) (The Economist, 2015)), en el sector de agua y saneamiento urbano (que es competencia de los Gobiernos Autónomos Descentralizados Municipales (GAD-M)), se ha convertido en una prioridad de las administraciones locales empeñadas en mejorar la calidad de vida de sus ciudadanos. En la actual coyuntura, las administraciones locales deben hacer frente a las inversiones en obra pública con sus propios recursos, es decir, sin mayores aportes no rembolsables (subvenciones) provenientes de las arcas centrales.

En este contexto, el volumen de inversión pública podría ser considerado un indicador clave en el marco de un modelo de desarrollo local, pues puede ser usado como una medida de la brecha que se necesita cubrir para alcanzar los niveles de cobertura que garanticen el nivel de bienestar y de derechos que la administración y las leyes establecen para los ciudadanos (Constitución de Ecuador, 2008, artículo 3, establece que es obligación del Estado garantizar el agua para sus habitantes, y el artículo 12, establece el derecho humano al agua.).

Con estos antecedentes, y en virtud de apoyar a los GAD municipales del Ecuador en la búsqueda de recursos financieros y de alternativas viables de co-financiación de obra pública en el sector de agua y saneamiento urbano, se plantea el presente trabajo, en el que se pretende ofrecer una panorámica concreta sobre las posibilidades que ofrecen las alternativas de alianzas estratégicas y que se ayude a los GAD municipales a cumplir con su mandato y en última instancia, contribuir a una mejora en la prestación de los servicios relacionados con los sectores de agua y saneamiento.

\section{Método de investigación}

El método de investigación utilizado consiste en el análisis de contenidos de lo que son las 
APP y de manera especial su aplicabilidad al sector de agua y saneamiento en Ecuador.

En primer lugar, se realizará un análisis normativo del sector agua y saneamiento y su viabilidad en Ecuador, y en segundo lugar, un análisis básico de los modelos de APP y su aplicabilidad.

\section{Análisis normativo}

En el tema normativo y sumado al panorama poco alentador en cuanto a disponibilidad de recursos propios para la financiación de obras públicas en el sector de agua y saneamiento con la que cuentan las administraciones locales (que son las competentes en este sector), la Constitución de la República (2008, art. 318), condiciona que la gestión y la prestación del agua sea exclusivamente de carácter público o comunitaria, contemplando que el servicio público de saneamiento, el abastecimiento de agua y el riego serán prestados únicamente por personas jurídicas estales o comunitarias, lo que limita la posibilidad de que otros sectores, y principalmente el sector privado, pueda participar de forma directa en la prestación de los servicios relacionados con el agua.

Esta limitación a la participación privada se fundamenta en la premisa de considerar el servicio público de saneamiento, el abastecimiento de agua potable y el riego, como sectores estratégicos. Si bien es cierto, estos sectores son de carácter estratégico, no es menos cierto que se restringe la participación del sector privado de manera independiente (mediante concesiones, por ejemplo), y no se permite a la población la posibilidad de contar con estos servicios en los casos que el sector público no pueda hacer frente a las inversiones requeridas. Sin embargo, al contemplar que personas juridicas estales, puedan participar en la prestación del servicio, se abre la posibilidad de que, por ejemplo, empresas mixtas (que son personas jurídicas estatales con capitales privados y públicos) puedan colaborar en este sector bajo modalidades de Alianzas Público-Privada (APP).

En el mismo sentido, la Ley Orgánica de Recursos Hídricos, Usos y Aprovechamiento del Agua LORHUyA (2014) limita de manera importante la posibilidad de encontrar alternativas de financiación de las obras en el sector, ya que amparada igualmente en el concepto de sector estratégico que tiene el agua, condiciona (prohíbe, art. 6) que la misma pueda ser prestada o gestionada por cualquier forma de administración que no sea la pública o comunitaria, con lo cual, se impide (salvo declaraciones de emergencia y subprocesos de carácter administrativo, art. 7), la participación de otros esquemas de gestión y administración de los servicios públicos, como son las APP y dentro de ellos las empresas de economía mixta que, por otro lado, están permitidas por la ley para otros sectores igualmente estratégicos para el país (Ley Orgánica de Empresas Públicas (LOEP, 2009, art. 35), pero lamentablemente se condiciona su participación en el sector de agua y saneamiento.

Afortunadamente, en el Reglamento de la Ley Orgánica de Recursos Hídricos, Usos y Aprovechamiento del Agua (expedida en abril del año 2015), y su reforma (expedida en agosto de 2015), en su artículo 6 señala que la iniciativa de la economía popular y solidaria, la iniciativa privada, o una empresa mixta en la que el Estado tenga mayoría accionaria podrán participar en determinados subprocesos de la administración del servicio público del agua que comprenden aquellas actividades relacionadas con la provisión de agua potable y saneamiento ambiental, cuando los GAD municipales no tengan las condiciones técnicas o financieras necesarias para hacerlo por si mismos. Con estas medidas de excepcionalidad se abre la puerta para la participación privada siempre y cuando los 
GAD justifiquen ante la autoridad del agua y más concretamente ante la Agencia de Regulación y Control del Agua (ARCA), la incapacidad técnica o financiera para abordar los subprocesos antes mencionados.

Adicionalmente, la interpretación de los artículos 315 (manifiesta que el Estado constituirá empresas públicas para la gestión de sectores estratégicos) y 316 (establece que el Estado podrá delegar la participación en sectores estratégicos y servicios públicos a empresa mixtas en las cuales tenga mayoría accionaria) de la Constitución de la República (2008), y el inciso segundo del artículo 4 de la Ley Orgánica de Empresas Públicas (LOEP, 2009) (que señala: "Las empresas subsidiarias son sociedades mercantiles de economía mixta creadas por la empresa pública, en las que el Estado o sus instituciones tengan la mayoría accionaria"), y por mandato del inciso segundo del artículo 282 del Código Orgánico de Organización Territorial, Administración y Descentralización (COOTAD, 2010) (que establece textualmente lo siguiente: "En las empresas de economía mixta en que participan los GAD, deberá el sector público poseer al menos el cincuenta y un por ciento del paquete accionario de la empresa"), abren también la posibilidad a la participación de las APP en el sector del agua y el saneamiento al señalar que el Estado podrá delegar la participación en los sectores estratégicos y servicios públicos a empresas mixtas en las cuales el sector público tenga mayoría accionaria (51\% de las acciones).

De igual manera, el mencionado artículo 316 manifiesta que de forma excepcional, se puede delegar a la iniciativa privada y a la economía popular y solidaria, el ejercicio de estas actividades, en los casos que establezca la ley; y de hecho, el Código de la Producción, Comercio e Inversiones ( 2010, art. 100) también dispone que en forma excepcional, debidamente decretada por el presi- dente de la república, cuando sea necesario y adecuado para satisfacer el interés público, colectivo o general, cuando no se tenga capacidad técnica o económica, o cuando la demanda del servicio no pueda ser cubierta por empresas públicas o mixtas, el Estado o sus instituciones podrán delegar a la iniciativa privada o a la economía popular y solidaria, la gestión de sectores estratégicos y la provisión de servicios públicos de electricidad, vialidad, infraestructuras portuarias o aeroportuarias, ferroviarias y "otras", dentro de las cuales se podría incluir al sector de agua y saneamiento. En el mismo artículo 100, se determina la modalidad de delegación, que podrá ser la concesión, alianza estratégica u otras formas contractuales de acuerdo a la ley.

Con estas consideraciones, el Gobierno del Ecuador ha emitido el Decreto Ejecutivo No. 582 del 18 de febrero de 2015, en el que se estableció el Reglamento del Régimen de Colaboración Público-Privada (2015), en el que se establecen las normas que regulen la asociación entre la entidad pública y las empresas privadas, como modalidad de delegación excepcional de cualquier proyecto en las áreas a que se refiere el Artículo 100 del Código Orgánico de la Producción, Comercio e Inversiones (2010). En el mencionado Decreto Ejecutivo No. 582 (art. 2), se establece que las iniciativas privadas pueden referirse a cualquier proyecto relacionado con sectores estratégicos, servicios públicos o cualquier otro servicio de interés general, tanto nuevos como aquellos existentes, respecto de los cuales exista contratos en ejecución.

Igualmente, el 18 de diciembre de 2015 se aprobó el proyecto de Ley Orgánica de Incentivos para las Asociaciones Público Privadas y la Inversión Extranjera (LOIAPP, 2015), que tiene por objeto gena erar incentivos principalmente de tipo tributario para fomentar las asociaciones público privadas, y atraer de esta manera a los inversionistas 
nacionales y extranjeros. Cabe mencionar, que en este caso también el sector del agua y saneamiento ha sido excluido de la LOIAPP, ya que en dicha Ley se manifiesta en su artículo 5 que los incentivos tributarios no aplican a servicios en los sectores estratégicos, es decir, que simplemente las iniciativas de APP en el sector del agua no podrán beneficiarse de los incentivos tributarios, pero se podrían implementar utilizando los mecanismos anteriormente analizados, principalmente, a través de las posibilidades que ofrece la Ley Orgánica de Empresas Públicas (LOEP, 2009). Según autores como Baldeón (2016), la LOIAPP no constituye una ley específica de APP que brinde certeza y seguridad jurídica, sino que más bien se centra en un marco jurídico para impulsar un "estado empresario" con una participación privada menor, lo que no debería ser el objetivo de una ley que pretenda ser atractiva para las inversiones privadas.

En tal sentido, se colige que una empresa pública en la que participe un GAD puede asociarse legalmente con una empresa privada, mediante la figura de "alianza estratégica" (que es la más recomendable, sobre todo si se incluyen mecanismos de transferencia tecnológica), a fin de generar otros emprendimientos, siempre que el objetivo de tal asociación se enmarque dentro de los fines para los cuales fue creada, los cuales constarán en el acto jurídico de creación que rige la vida jurídica de cada empresa, así como de las competencias exclusivas que les corresponden a los $G A D$, según la Constitución de la República de 2008, y en concordancia con el Código Orgánico de Organización Territorial y Descentralización (COOTAD, 2010).

Recientemente, y al amparo de la Ley para el Fomento Productivo, vigente desde el 21 de agosto de 2019, el gobierno ha anunciado que plantearán reformas al modelo de Alianzas Público-Privadas en el país, dado el escaso éxito que la misma ha alcanzado en Ecuador en comparación con las inversiones realizadas en otros países de la región (El Universo, 2009). El Banco Mundial ha estimado que en Ecuador, en 10 años que lleva analizando este mecanismo de inversiones, ha logrado implementar únicamente cinco proyectos importantes con una inversión estimada en USD 500 millones (0,07 \% del Producto Interno Bruto -PIB-), mientras que países como Brasil, en el mismo período, ha concretado inversiones por alrededor de USD 230.800 millones de dólares (505 proyectos, $1.05 \%$ del PIB), México por un monto de alrededor de USD 40.400 millones (120 proyectos, 0,37\% del PIB), y Colombia por un monto de USD 25.500 millones (58 proyectos, 0,85\% del PIB). Ecuador en temas de inversiones en APP, si tenemos en cuenta, el monto está a la par de Belice (2 proyectos, $0,81 \%$ del PIB) y Haití (2 proyectos, 0,16 \% del PIB) con alrededor de USD 100 millones, o de El Salvador (5 proyectos, 0,07 \% del PIB) con alrededor de USD 150 millones (Banco Mundial, 2017).

Es decir, a pesar de contar con la legislación para promover las APP, en Ecuador se ha presentado un desempeño muy pobre en todos los sectores en la implementación de esta modalidad de inversión (500 millones de dólares en los últimos 10 años). Y si se toma en cuenta las mayores restricciones que se han detallado, que presenta el sector de agua y saneamiento, que, por la característica de estratégico del recurso, el panorama para dicho sector es aún menos halagador.

\section{Aplicabilidad de las APP}

En el segundo aspecto a analizar sobre las APP y su aplicabilidad resulta interesante conocer las definiciones y características principales de las APP y sus ejemplos emblemáticos, en particular, a nivel latinoamericano, las mismas que se pueden encontrar en 
trabajos desarrollados por el Banco de Desarrollo de América Latina (2015) (CAF, 2010). A nivel del sector agua, el análisis sobre la importancia de la participación privada mediante esquemas de APP, a nivel igualmente regional, se pueden encontrar en trabajos desarrollados por el Banco Interamericano de Desarrollo (2006) (CAF, 2015), y otros autores como Baldeón (2016), Chafla y Cerón (2016), CONGOPE (2017) y Chafla (2017), que lo analizan para el caso del Ecuador.

En tal sentido, los beneficios o inconvenientes de estos modelos de APP, como elemento viable y actual para la co-financiación de la obra pública local ya han sido ampliamente analizados, considerándose suficiente en esta investigación realizar simplemente algunas precisiones de estos esquemas para el caso de sector agua y saneamiento.

En primer lugar, es necesario desligar la idea de que las APP implican necesariamente la privatización, ya que dicha posibilidad para el caso del sector agua y saneamiento está prohibida en la Constitución de la República del Ecuador (art. 318). Los modelos APP (incluso en las concesiones), el sector público mantiene la titularidad de las infraestructuras y conserva (por contrato), todas las facultades de control necesarios para garantizar el buen funcionamiento del servicio y los derechos de los ciudadanos.

En tal sentido, resulta interesante concretar lo que no se debería entender por un modelo de APP en términos generales:

- No significa la privatización de los recursos y los servicios públicos.

- No significa que únicamente pueden operar empresas extranjeras (está abierto a todas las empresas que cuenten con las garantías y experiencia necesarias).

- No significa que se va a despedir, necesariamente, a los empleados públicos (aunque es posible que se rea- licen procesos de optimización del personal).

- No significa que los servicios van a costar necesariamente mucho más que si lo hace una empresa pública (pueden incluso ser menos costosos si se aplican criterios de eficiencia y optimización del gasto).

- No significa que el sector público sea ineficiente per se, y que no cuente con profesionales competentes que puedan participar en la dotación de los servicios, sino que el sector público está caracterizado por rigideces (salariales, administrativas, burocráticas, etc.) que lo hacen menos ágil.

Una vez que se ha detallado lo que no se debería entenderse como una APP resulta conveniente presentar algunos lineamientos que nos ayuden a entender de mejor manera lo que debería ser una APP. Las APP se pueden caracterizar en cuatro ideas básicas (CAF, 2015):

1. Existe una serie de bienes y servicios de interés general, que, debido a los fallos de mercado, como monopolios naturales, bienes públicos o presencia de externalidades, deben ser supervisados por el sector público.

2. El sector privado puede contribuir significativamente a una mayor eficiencia y calidad en la asignación de los recursos públicos.

3. Existe la necesidad de definir una adecuada distribución de riesgos entre el sector público y el sector privado en la dotación de bienes y servicios públicos.

4. La participación del sector privado en todas las fases del ciclo de proyecto (diseño, construcción, financiación, mantenimiento y operación) puede beneficiar la provisión de bienes y servicios públicos. 
En un modelo de co-financiación de obra pública como son las APP, el sector privado comparte el riesgo con la administración pública, pero la titularidad (propiedad) de las infraestructuras siempre será de la administración local. A parte de compartir los riesgos financieros de las inversiones, lo que resulta significativo en los modelos de APP es que se comparten experiencias, el saberhacer (know how), que poseen las empresas privadas de trayectoria consolidada, para mejorar la calidad de los servicios públicos y de esta manera elevar la eficiencia en la gestión de los recursos públicos, lo que repercutirá significativamente en mejorar la calidad de vida y el bienestar de los ciudadanos.

Por otro lado, hay que tener presente, que la realidad ha demostrado que en el país son muy pocos los proyectos de agua $y$, principalmente, de saneamiento (en el que se incluyen alcantarillado sanitario, pluvial, depuración de aguas residuales), que puedan ejecutarse sin aportaciones públicas, lo que puede verificarse por la necesaria intervención del Gobierno central, vía créditos reembolsables y no reembolsables otorgados por el Banco de Desarrollo del Ecuador (BdE). En tal sentido, los esquemas de APP, bien estructurados, pueden ser la contraparte necesaria a las aportaciones públicas (cada vez más escasas) para que estos proyectos puedan implementarse y no se prive a los ciudadanos de las infraestructuras necesarias por la dotación de los servicios de agua y saneamiento.

De igual manera, hay que tener claro que para la implementación de estos esquemas en Ecuador es fundamental analizar y solventar primero varios problemas de tipo estructural tales como: el contar con un recurso humano especializado no solo en la estructuración de proyectos bajo la modalidad de APP, sino también en el diseño, implementación y evaluación de este tipo de proyectos. Por otro lado, es necesario contar con una legislación que defina claramente las características de los contratos a firmar entre las partes, la distribución de los riesgos, las garantías de calidad en la dotación de los servicios y la adecuada resolución de las controversias y conflictos. En este caso, se puede citar a manera de ejemplo, la "Ley Marco de Asociaciones Público Privadas para la generación de empleo productivo y dicta normas para la agilización de los procesos de promoción privada" del Perú (Ley Marco de Asociaciones Público Privadas, 2008) y su Reglamento del Decreto Ejecutivo No. 1022, las cuales podrían aportar elementos importantes para favorecer la aplicabilidad de estos esquemas en Ecuador, dado que es una normativa que lleva funcionando un tiempo prudencial y se han podido ir puliendo las posibles inconsistencias. En la actualidad, el monto de inversiones en APP en Perú alcanza los USD 23.400 millones en 80 proyectos que constituyen el 1,53 \% de su PIB (Banco Mundial, 2017).

\section{Resultados}

A la luz de lo expuesto, es posible presentar un primer resultado en relación con la posibilidad real de implementar un modelo de APP en el país para el sector del agua y saneamiento que es el objetivo central que ha guiado la presente investigación. La participación de la iniciativa privada, y por tanto los esquemas de APP, sería factible en el sector agua y saneamiento en el Ecuador, básicamente mediante una figura de "excepcionalidad" contemplada en el Reglamento de la LORHUyA y sustentada en la incapacidad técnica y/o económica de los GAD municipales quienes son los competentes para la dotación de estos servicios públicos. No obstante, hay que tener presente el costo de tipo político que puede suponer a un GAD-M declarar tal situación de incapacidad principalmente técnica, la misma que 
igualmente debería ser demostrada por la falta de recursos técnicos y humanos de las administraciones locales, lo que dificultaría grandemente la implementación de este mecanismo. También habría que considerar el posible riesgo de que dicho Reglamento pueda ser modificado mediante un Decreto Ejecutivo, lo que podría reinterpretar la Ley y cambiar estas condiciones.

Tal y como se ha presentado la situación actual del sector de agua y saneamiento en Ecuador, nos encontramos en un escenario de escases de recursos para la financiación de obra pública, agravada por una restricción legislativa que no facilita la implementación de otras alternativas de financiación, con lo cual se limita el horizonte de posibilidades de elección dando como resultando un modelo ineficiente desde el punto de vista económico (no se puede elegir el esquema que maximice el bienestar social), y sobre todo no ayuda a resolver el problema de déficit en la dotación de los servicios de agua y saneamiento, que para el año 2015 se estimaba un nivel de cobertura para todo el Ecuador de alrededor del $77,3 \%$ para agua potable y $66,3 \%$ para el servicio de alcantarillado (Senagua, 201\%). Solamente el 85,9\% de la población a nivel nacional cuenta con saneamiento básico y el 85,5\% cuenta con instalaciones para el lavado de manos con agua y jabón, y el 5,7 \% de la población en el sector rural no dispone de servicios higiénicos para realizar sus necesidades (INEC, 2017).

La brecha que hay que cubrir en cuanto a población urbana con acceso a fuentes mejoradas (no totalmente seguras) de abastecimiento de agua potable, se estimó para Ecuador en 2015 en torno al 15 \% (el 85 \% tiene acceso a fuentes mejoradas), lo cual si bien es significativo aún no se llega a cubrir el $100 \%$ de la población como ya lo han alcanzado otros países Latinoamericanos como es el caso de Uruguay, Paraguay, Chile, Costa Rica, y Brasil (CAF y BID, 2015).

En tal sentido, las APP podrían ser utilizadas como un indicador en favor de la reducción de estas brechas de inversiones en infraestructuras para la dotación de servicios básicos, ya que a mayores montos de inversión alcanzados por los países en estos esquemas de co-financiación, darán como resultado menores brechas de cobertura que se tenga en servicio básicos y de esta manera, más cerca se estará de alcanzar los niveles de bienestar que requieren los ciudadanos. Desafíos como los que nos marcan, por ejemplo, los Objetivos de Desarrollo Sostenible (ODS), y el objetivo 6 (garantizar la disponibilidad de agua y su gestión sostenible y el saneamiento para todos en el año 2030), se podrán alcanzar con mayor facilidad si se cuenta con adecuado apoyo del sector privado apoyando en la financiación de estas infraestructuras.

La contribución de los beneficiarios (consumidores) de estos servicios públicos para la recuperación de los costos que generan es una necesidad ineludible en la actualidad. Las tarifas que se pagan por los servicios de agua y saneamiento no pueden ser tarifas únicamente de sostenibilidad financiera que sirvan en el mejor de los casos para cubrir los costos de operación y mantenimiento de las infraestructuras, sino que en la medida de lo posible, deben también ayudar a cubrir los costos de las inversiones. Tarifas realistas para los servicios públicos que reflejen los verdaderos costos de los servicios, y que ayuden a racionalizar y a hacer eficiente el consumo de un recurso escaso como es el agua, es una tarea ineludible.

Si bien es cierto, que los postulados de equidad, redistribución y justicia que deben guiar la determinación de cualquier tarifa de un servicio público no se pueden descuidar en este análisis, la necesidad de que los servicios se paguen vía tarifas por parte de los beneficiarios, y no vía subvenciones en la 
que la pagan todos los ciudadanos independientemente de su consumo a través de sus impuestos resulta en un mecanismo eficiente y equitativo de gestión de recursos escasos en la economía.

\section{Referencias}

Banco de Desarrollo de América Latina (CAF). (2015). Asociación Público Privada en América Latina. Aprendiendo de la experiencia. Corporación Andina de Fomento Vicepresidencia de Infraestructura.

Baldeón, I. (2016). Las asociaciones público privadas (APP) en el marco jurídico ecuatoriano. Foro: Revista de Derecho. 25: 101-124.

Banco Mundial (BM). (2017). Una década de alianzas público-privadas en América Latina y el Caribe. Disponible en https://blogs. worldbank.org/es/ppps/una-d-cada-dealianzas-p-blico-privadas-en-am-rica-latina-y-el-caribe-qu-hemos-aprendido

Banco de Desarrollo de América Latina (CAF). (2010). Infraestructura Pública y Participación Privada: conceptos y experiencias en América y España. Corporación Andina de Fomento Vicepresidencia de Infraestructura.

Banco de Desarrollo de América Latina (CAF) y Banco Interamericano de Desarrollo (BID). (2015). El futuro de los servicios de agua y saneamiento en América Latina. Documento para discusión. 2015.

Chafla, P. (2017). APP, Alternativa para la co-financiación de obras de agua y saneamiento en Ecuador. Revista lagua. 15. https:// www.iagua.es/blogs/pablo-chafla/alianzas-publico-privadas-alternativa-viablesector-agua-ecuador.

Chafla, P. y Cerón, P. (2016). Esquemas de Participación Público-Privada en el sector del agua y saneamiento en Latinoamérica. Revista Tecnología y Ciencias del Agua. México //(3), 5-17. Ley Marco de Asociaciones Público Privadas para la generación de empleo productivo y dicta normas para la agilización de los procesos de promoción privada del Perú. (2008). Diario Oficial El Peruano. 13 de mayo.
Consorcio de Gobiernos Autónomos Provinciales del Ecuador (CONGOPE). (2017). Alianzas Público-Privadas y Desarrollo Local. Abya-Yala.

Constitución de la República del Ecuador. 2008. Decreto Legislativo 0. Registro Oficial 449, 29 de octubre.

Instituto Nacional de Estadísticas y Censos (INEC). (2017). Medición de los indicadores ODS de Agua, Saneamiento e Higiene (ASH) en el Ecuador.

Código Orgánico de Organización Territorial, Administración y Descentralización (COOTAD). (2010). Registro Oficial Suplemento 303 de 19 de octubre.

Código Orgánico de la Producción, Comercio e Inversiones. (2010). Registro Oficial, Año II, Quito, miércoles, 29 de diciembre, N. 351.

Decreto Presidencial No. 582. Reglamento del régimen de colaboración público-privada. Registro Oficial N. ${ }^{0} 453$ de 6 de marzo del 2015.

El Universo (2019). Secuela de ley abordará alianzas público-privadas. El Universo, 29 de enero. Disponible en https://www. eluniverso.com/noticias/2019/01/29/ nota/7162511/secuela-ley-abordaraalianzas-publico-privadas

Inter-American Development Bank. (2006). Financial Structuring of Infrastructure Projects in Public-Private Partnerships: An Application to Water Projects. Washington.

Ley Orgánica de Incentivos para Asociaciones Público-Privadas y la Inversión Extranjera. (2015). Registro Oficial Año III, N. ${ }^{0} 652$ del 18 de diciembre.

Ley Orgánica de Recursos Hídricos, Usos y Aprovechamiento del Agua (LORHUyA). (2014). Registro Oficial, Año II - N. . 305. 6 de agosto.

Ley Orgánica de Empresas Públicas (LOEP). (2009). Registro Oficial Suplemento 48 del 16 de octubre.

Reglamento a la Ley Orgánica de Recursos Hídricos, Usos y Aprovechamiento del Agua. Primer Suplemento del Registro Oficial 483 del 20 de abril de 2015.

Reformas al Reglamento a la Ley Orgánica de Recursos Hídricos, Usos y Aprovechamiento del Agua. (2015). Registro Oficial 570, 21 de agosto. 
Reglamento del Decreto Legislativo N. ${ }^{0} 1012$ que aprueba la Ley Marco de Asociaciones Público Privadas para la generación de empleo productivo y dicta normas para la agilización de los procesos de promoción privada del Perú. Publicado en el diario oficial El Peruano, 31 de mayo de 2014. Secretaría del Agua (Senagua). (2015). Presentación Institucional.

The Economist (2015). Perspectivas sobre infraestructuras urbanas 2015. The Economist Intelligence Unit. Patrocinado por FCC. 